\title{
XUV laser mass spectrometry for nano-scale 3D elemental profiling of functional thin films
}

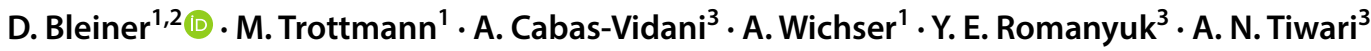

Received: 14 October 2019 / Accepted: 4 February 2020 / Published online: 26 February 2020

(c) The Author(s) 2020

\begin{abstract}
Direct nano-scale microanalysis is important for photovoltaic functional thin films to characterize their homogeneity and purity. This demands combining spatial resolution in the micro/nano-scale and sensitivity in the trace-level range, which is at the moment beyond state-of-the-art. As dictated by counting statistics, the reduction of the spot size degrades the detection limit. The utilization of a tabletop XUV laser at $\lambda=46.9 \mathrm{~nm}$ has shown to dramatically improve the ablation efficiency with respect to that of visible lasers, such that ablation spot of $1 \mu \mathrm{m}$ limits. Li-doped $\mathrm{Cu}_{2} \mathrm{ZnSn}(\mathrm{S}, \mathrm{Se})_{4}$ (so-called kesterite) thin films were irradiated across 3D ablation arrays for hyperspectral mapping by means of time-of-flight mass spectrometry. The nominal 3D data node lattices were the initialisation perceptron, filled with measured values, and for a detailed supervised learning postprocessing, the node-to-node links were analysed by means of a 2D-kernel covariance algorithm. The latter permitted to obtain robust 3D elemental distribution functions well below the measurement spacing, giving insights into the inhomogeneity and impurities.
\end{abstract}

Keywords XUV Laser $\cdot$ Plasma $\cdot$ Photovoltaic thin films $\cdot$ Depth profiling $\cdot$ Capillary discharge $\cdot$ Chemical mapping

\section{Introduction}

Direct solid-sample microanalysis, by means of laser ablation, expands the capabilities of traditional chemical analysis in two directions: (1) materials can be directly analyzed "as they are", with no preparation; (2) chemical information is obtained in a space-resolved way at micro-scale. The former is beneficial in terms of (1) speed of the analysis, (2) minimization of potential artifacts due to the direct interrogation without intermediate steps, (3) possibility to measure composition in the "natural matrix", i.e. the material does

D. Bleiner: Invited Lecture at the COLA Conference 2019 in Maui, HI, USA.

D. Bleiner

davide.bleiner@empa.ch

1 Laboratory for Advanced Analytical Technologies (Empa Materials Science and Technology), Überlandstrasse 129, 8600 Dübendorf, Switzerland

2 Department of Chemistry, University of Zurich, Winterthurerstrasse 190, 8057 Zurich, Switzerland

3 Laboratory for Thin Films and Photovoltaics (Empa Materials Science and Technology), Überlandstrasse 129, 8600 Dübendorf, Switzerland not have to be pre-treated. In some cases, e.g. ceramics, fly ashes, etc. the matrix is so hard that classical wet chemical attack is not possible.

State-of-the-art laser ablation has, however, a few limitations that may be addressed in the near future, thanks to shorter wavelengths $[1,2]$. First, the ablation mechanism is associated with thermo-mechanical processes that may modify the material composition, or damage a large portion of it. Use of ultrashort pulses has shown benefit for conductors, but not so extensively for dielectric materials [3]. Second, the spatial resolution is limited by the diffraction limit, such that the downscaling of the spot requires shorter wavelength lasers. However, a reduction of the sampled mass degrades the sensitivity as dictated by counting statistics.

Thin films of solution-processed kesterite have been investigated as a material for efficient photovoltaic (PV) modules. The matrix is composed of $\mathrm{Cu}, \mathrm{Zn}, \mathrm{Sn}, \mathrm{S}$ and $\mathrm{Se}$ (CZTSSe), whose doping with alkali elements enhances the PV efficiency. Interestingly, extensive studies showed that only lithium (Li) can alloy to the absorber layer, and provides the highest device performance boost compared to the other alkali elements [4-6].

Kesterite shares a similar crystal structure with other thinfilm chalcogenide solar cell technologies, such as CdTe and 
$\mathrm{Cu}(\mathrm{In}, \mathrm{Ga})(\mathrm{S}, \mathrm{Se})_{2}(\mathrm{CIGSSe})$. However, contrary to the latter ones, kesterite is based on earth-abundant and low-toxic elements. Nevertheless, the CZTSSe record efficiency of $12.6 \%$ falls far below the Shockley-Queisser limit of 30\% [7], whereas both CdTe and CIGSSe technologies have reached efficiencies above 20\% [8]. Kesterite progress is possibly hampered by its narrow chemical stability region [9], which leads to the formation of undesired secondary and ternary phases during the annealing process [10]. Henceforth, the chemical composition is inhomogeneous throughout the absorber thickness.

To investigate the composition of the thin films, characterization at low and large scale in 3D is essential. In particular, the compositional homogeneity as well as the purity of the material is of concern. A number of "cross-sectional" techniques are available to perform surface ( $x$ by $y$ ) or depth ( $x$ by $z$ ) mappings. The strength of laser ablation, however, lies in the ability of 3D mapping by repeated pulse delivery ( $z$-dimension) while the sample stage is scanned ( $x$ - and $y$-dimension). The ablated mass is analyzed online by mass spectrometry to obtain rapid insights into the composition.

As discussed above, the ablation mechanism changes radically while using a visible or a short-wavelength laser, which has an impact on the chemical data. Aim of this work was to compare the performance of traditional visible laser ablation microanalysis with more advanced short-wavelength (XUV) laser ablation, on the case of CZTS functional thin films for PV. Previous studies on water-oxidation catalysis showed a remarkable impact in mapping rapidly the 3D chemical and mechanical modifications of a sample cycled in the electrocatalytic system 1 [1]. The sample did not lose its functionality, such that it is in principle possible to deploy XUV-MS for real-time operando spectroscopy. Bleiner et al. have recently reviewed the field and provided a comprehensive tutorial for a general understanding. ${ }^{1}$

\section{Experimental}

\subsection{Photovoltaic thin-film samples}

The precursor solution consisted of thiourea $(99 \%+$, SigmaAldrich), tin chloride dihydrate $\left(\mathrm{SnCl}_{2} \cdot 2 \mathrm{H}_{2} \mathrm{O}, 98 \%\right.$, SigmaAldrich), zinc chloride $\left(\mathrm{ZnCl}_{2}, 99.99 \%\right.$, Alfa Aesar), copper chloride dihydrate $\left(\mathrm{CuCl}_{2} \cdot 2 \mathrm{H}_{2} \mathrm{O}, \geq 99.99 \%\right.$, Sigma-Aldrich) and lithium chloride anhydrous ( $\mathrm{LiCl}, 99 \%$, Fluka) dissolved in dimethyl sulfoxide (DMSO, 99.9\%, Alfa Aesar). A $200-300 \mathrm{~nm}$ thick $\mathrm{SiO}_{x}$ alkali diffusion barrier layer was sputtered onto a $1 \mathrm{~mm}$ thick soda lime glass (SLG) with a

\footnotetext{
1 The paper is under advanced review and the reference will be added at the time of publication of the present article.
}

subsequent deposition of $1 \mu \mathrm{m}$ of molybdenum. The precursor solution was spin coated onto the Mo layer and dried on a hotplate at $320^{\circ} \mathrm{C}$ in air. The spin-coating and drying steps were repeated 12 times to obtain the desired precursor film thickness of $1.5 \mu \mathrm{m}$. The sample was annealed in a rapid thermal processing furnace (RTP Annealsys AS ONE 150) inside a closed graphite box with selenium pellets $(800 \mathrm{mg}$ ). The temperature gradient employed for annealing was the three-stage process with holding at 300,500 , and $550{ }^{\circ} \mathrm{C}$.

\subsection{Electron microscopy}

The images were taken on a FEI Nano SEM 230 (SEM) and a FEI, Helios 660 G3 UC (FIB/SEM). The cross-section of the XUV ablation crater has been cut and polished at an ion energy of $30 \mathrm{kv}$ and ion currents ranging from $47 \mathrm{nA}$ to $780 \mathrm{pA}$. To prevent the surface from severe damage, a sacrificial layer of tungsten has been deposited by means of ion-induced deposition.

\subsection{Optical laser ablation with ICP-MS}

Laser ablation (LA) inductively coupled plasma mass spectrometry (ICP-MS) measurements were conducted using a PerkinElmer NexION2000 ICP-MS integrated with an optical laser ablation system developed in-house. The laser system consists of a $10 \mathrm{~Hz} \mathrm{Nd:YAG} \mathrm{laser,} \mathrm{2nd} \mathrm{harmonics,}$ $6 \mathrm{~ns}$ pulse length, a focusing and positioning optic and an automated $x y z$ sample table. The laser fluence is adjustable with a neutral density filter and Q-switch adaptation. For the pulse number, the probe position and the synchronization with the ICP-MS are controlled by a LabVIEW interface. The samples are positioned in an airtight laser cell $\left(2 \mathrm{~cm}^{3}\right)$ on the $x y z$ stage. By means of an aspherical lens, the laser beam is focused on the surface of the sample. The ablated sample material is transported directly by argon carrier gas into the ICP-MS. Typical operation parameters for optical laser ablation analysis are given in Table 1.

\subsection{XUV laser ablation mass spectrometry}

If LA in the visible generates a dry aerosol for ICP-MS analysis, the reduction of the wavelength has two advantages: (1) smaller spot and depth scales; (2) direct surface ionization, without requirement for the ICP ionization source. An Ar capillary discharge XUV laser $(\lambda=46.9 \mathrm{~nm}$ or $26 \mathrm{eV})$ generated pulses of about $10 \mu \mathrm{J}$ with duration of about $2 \mathrm{~ns}$ (FWHM) and a pulse to pulse energy fluctuation $<10 \%$. Grazing incidence optics were used to collimate the XUV laser and focus it on the sample using a zone plate. The ablated ions were energy homogenized to $1.5 \mathrm{keV}$, using the potential difference between the sample and the zone plate, which were set $2.1 \mathrm{~mm}$ apart. The zone plate had a 
Table 1 Optical laser ablation operating conditions used in this work

\begin{tabular}{l} 
Laser ablation parameters \\
Laser ablation system: Nd:YAG laser \\
Wavelength: $532 \mathrm{~nm}$ \\
Pulse duration: $6 \mathrm{~ns}$ \\
Laser energy: $6 \mathrm{~mJ}$ \\
Repetition rate: $10 \mathrm{~Hz}$ \\
Spot size: $100 \mu \mathrm{m}$ \\
Carrier gas (Ar) flow rate: $0.9-1.1 \mathrm{~L} \mathrm{~min}-1$ \\
ICP-MS conditions \\
ICP-MS instrument: NexION2000 \\
RF power: $1400 \mathrm{~W}$ \\
Auxiliary gas (Ar) flow rate: $1.2 \mathrm{~L} \mathrm{~min}{ }^{-1}$ \\
Plasma gas (Ar) flow rate: $15 \mathrm{~L} \mathrm{~min}{ }^{-1}$ \\
Dwell time per isotope: $0.5 \mathrm{~ms}$ \\
Detector mode: dual \\
Measured isotopes: ${ }^{7} \mathrm{Li} ;{ }^{13} \mathrm{C} ;{ }^{23} \mathrm{Na} ;{ }^{29} \mathrm{Si} ;{ }^{34} \mathrm{~S} ;{ }^{82} \mathrm{Se} ;{ }^{85} \mathrm{Ku} ;{ }^{88} \mathrm{Sr} ;{ }^{48} \mathrm{Ma} ;{ }^{118} \mathrm{Se} ;{ }^{63} \mathrm{Cu}$; \\
\hline
\end{tabular}

central opening of $50 \mu \mathrm{m}$ to allow the ions to be extracted to the time-of-flight mass analyzer, equipped with a dual micro-channel plate detector (chevron configuration), with $40 \mathrm{~mm}$ in diameter active area. The entire system occupies a footprint of $0.6 \times 3 \mathrm{~m}$. The mass spectra are processed and stored by a PC digitizer $\left(\mathrm{GaGe}^{\circledR}\right.$ EON CS121G1, 12 bits, $1 \mathrm{GS} / \mathrm{s})$. With $50 \Omega$ input impedance, the amplitude is limited to $\pm 5 \mathrm{~V}$. A typical full mass range TOF spectrum is $50 \mu \mathrm{s}$ (ca. up to $300 \mathrm{~m} / \mathrm{z}$ ) that permitted to obtain the complete elemental coverage.

\section{Results and discussion}

\subsection{Spatial resolution}

The laser pulse in the visible region produced a strong surface impact on the CZTSSe layer with a crater of $>400 \mu \mathrm{m}$ in diameter (Fig. 1) at an ablation rate $>400 \mathrm{~nm}$ per pulse, due to the soft material's characteristics. If a lower fluence would improve the space resolution, one must bear in mind that the detection of (thermo-mechanically) ablated species is prone to fractionation at low fluence and small spot. At high fluence, the CZTSSe layer showed an irregular detachment. With $n=5$ pulses (i.e. half a second at $10 \mathrm{~Hz}$ ), the entire layer pack was ablated across. The transient analyte signals had a spiky profile, which indicates micron-sized particles carrying agglomerated nano-particles on their surface, suddenly vaporized en masse as entering the plasma. All elements of the CZTSSe layer could be detected, though the quadrupole peak hopping method imposed to select ten isotopes (Table 2). The limit of detection of $\mathrm{Li}$ was determined as low as $1.3 \mathrm{mg} / \mathrm{kg}$. All other elements were also in

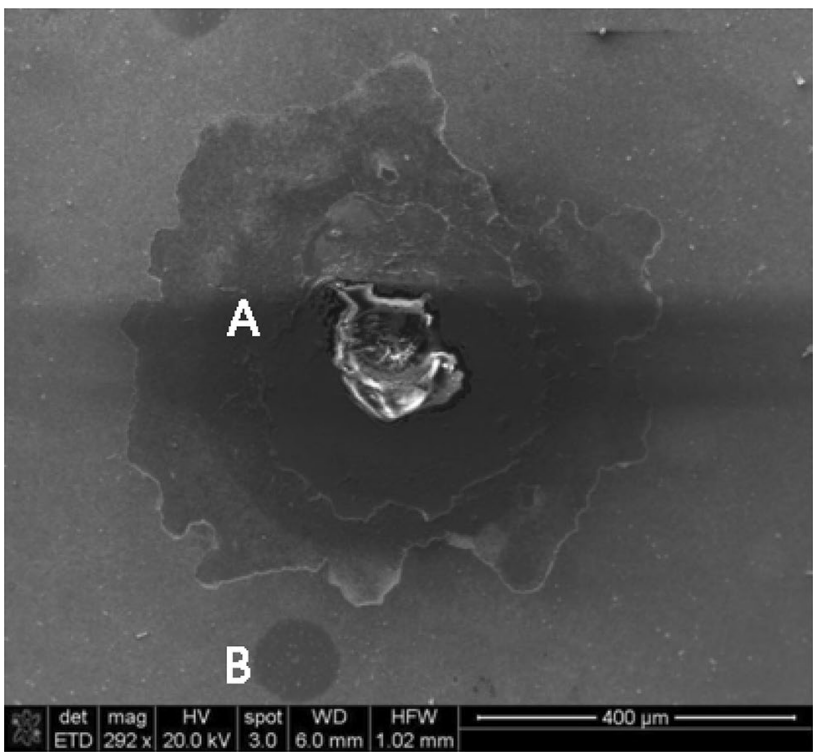

Fig. 1 Electron micrograph showing a comparison of ablation craters from laser irradiating the kesterite sample, i.e. using the visible laser (a) or the XUV laser (b). See text for details

Table 2 Optical laser ablation results

\begin{tabular}{llcrr}
\hline Isotope & Element & Signal (median) & Noise & \multicolumn{1}{c}{$\mathrm{S} / \mathrm{N}$} \\
\hline 7 & $\mathrm{Li}$ & 248.1 & 0.5 & 531.6 \\
13 & $\mathrm{C}$ & 444.2 & 394.4 & 1.1 \\
23 & $\mathrm{Na}$ & 9047.2 & 2.6 & 3479.7 \\
34 & $\mathrm{~S}$ & 125.0 & 110.6 & 1.1 \\
39 & $\mathrm{~K}$ & 1367.1 & 682.8 & 2.0 \\
63 & $\mathrm{Cu}$ & 5037.5 & 1.3 & 3778.1 \\
66 & $\mathrm{Zn}$ & 1269.6 & 0.9 & 1464.9 \\
82 & $\mathrm{Se}$ & 945.0 & 3.5 & 270.0 \\
85 & $\mathrm{Rb}$ & 4.0 & 0.8 & 4.8 \\
118 & $\mathrm{Sn}$ & 9874.6 & 1.3 & 7405.9 \\
\hline
\end{tabular}

It should be noted that the MS is a quadrupole, so the element menu was restricted to ten isotopes, which is different from the case shown with XUV laser ablation where a time-of-flight was used, which collects the full mass range (see Table 3). The median of the signal is shown, because of the mentioned spikes (see text), which by the average would generate artefacts. Since optical laser ablation is an atmospheric plasma ionization (API) technique, high blank values on $\mathrm{C}$ are common

the low ppm range. With sufficient measuring points, the optical laser ablation method can be used to qualitatively fingerprint dopants as well as impurities in the layers to accumulate counts, but the rapid destruction of the material thickness is limiting. Therefore, although the sensitivity is in principle, high, low mass availability as well as transport losses limit the end figures. 
Fig. 2 Average mass spectra at various depths by repeated pulse delivery on-spot, from surface (top) towards bulk (bottom). In the top panes, one notes the fine $3 \mathrm{~nm}$ Pt capping $(\mathrm{m} / \mathrm{z}, 194,195,196)$ that then is ablated through. Other peaks are from the main elements of the CZTSSe system $(\mathrm{Cu}$, $\mathrm{Zn}, \mathrm{Sn}, \mathrm{S}, \mathrm{Se})$ as well as the alkali-doping (Li), the soda lime glass substrate contaminants. Ultratrace contaminants of $\mathrm{W}$, $\mathrm{Au}$ and $\mathrm{Hg}$ are also found, not visible at this zoom (see text)
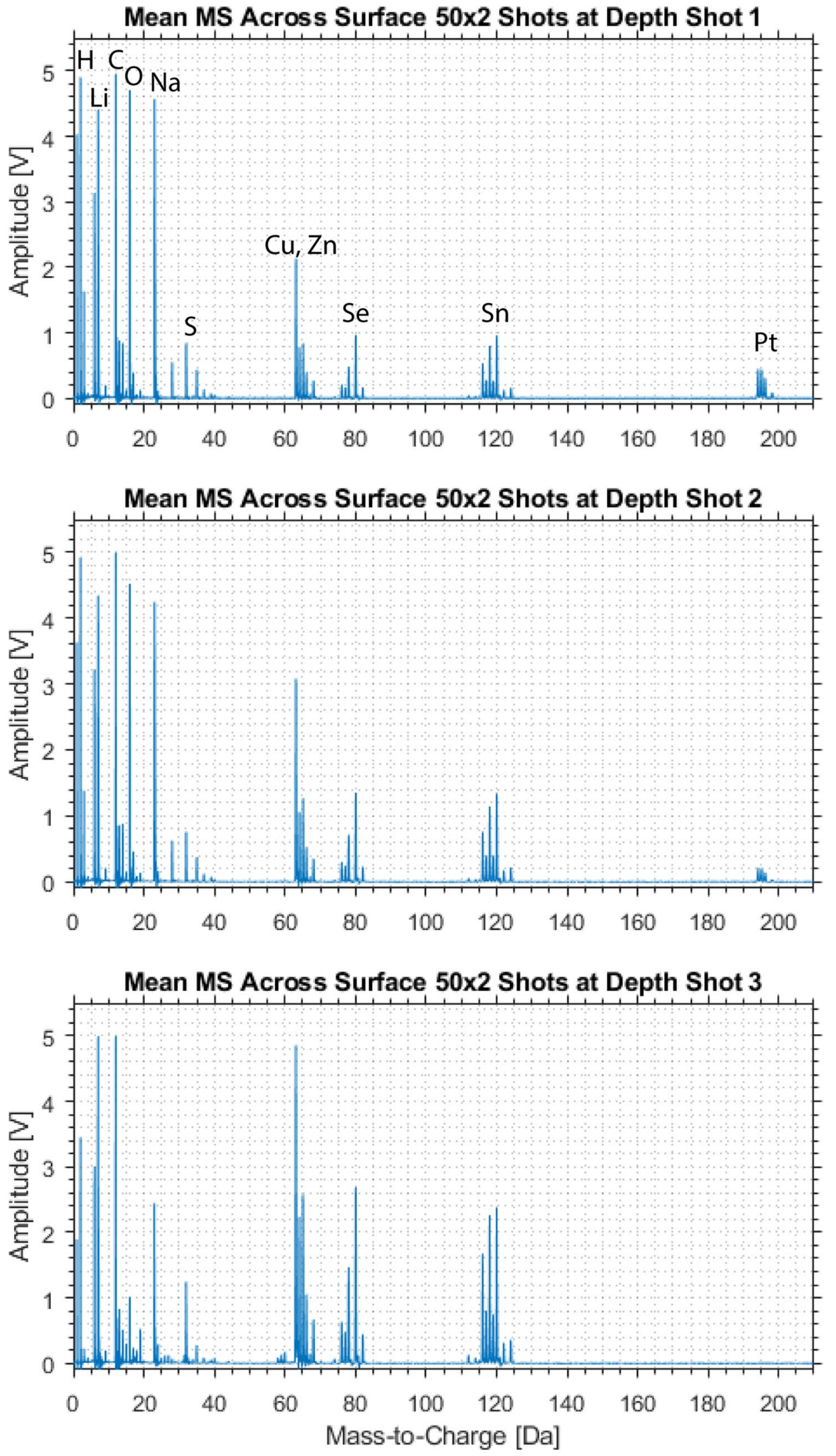
Table 3 Experimental (XUV laser ablation) and theoretical isotopic abundances for $\mathrm{Zn}$ and $\mathrm{Se}$, and related deviation

\begin{tabular}{|c|c|c|c|c|}
\hline Isotope & Experimental (\%) & Theoretical (\%) & Deviation (\%) & $\begin{array}{l}\text { Rate of doubly } \\
\text { charged (\%) }\end{array}$ \\
\hline \multicolumn{5}{|l|}{$\mathrm{Cu}$} \\
\hline 63 & 72.4 & 69.2 & -3.19 & 0.3 \\
\hline 65 & 27.6 & 30.8 & 3.19 & 1.2 \\
\hline Total & $100.0 \%$ & 100.0 & $<$ & \\
\hline \multicolumn{5}{|l|}{$\mathrm{Zn}$} \\
\hline 64 & 55.4 & 48.6 & -6.84 & \\
\hline 66 & 24.3 & 27.9 & 3.63 & \\
\hline 67 & 3.4 & 4.1 & 0.69 & \\
\hline 68 & 16.3 & 18.8 & 2.45 & \\
\hline 70 & 0.5 & 0.6 & 0.07 & \\
\hline Total & 100.0 & 100.0 & $<$ & \\
\hline \multicolumn{5}{|l|}{$\mathrm{Se}$} \\
\hline 74 & 1.0 & 0.9 & -0.08 & \\
\hline 76 & 10.9 & 9.4 & -1.53 & 1.0 \\
\hline 77 & 8.2 & 7.6 & -0.56 & \\
\hline 78 & 25.0 & 23.8 & -1.21 & 3.7 \\
\hline 80 & 47.3 & 49.6 & 2.27 & 1.2 \\
\hline 82 & 7.6 & 8.7 & 1.10 & \\
\hline Total & 100.0 & 100 & $<$ & \\
\hline \multicolumn{5}{|l|}{ Sn } \\
\hline 112 & 1.2 & 1.0 & -0.2 & \\
\hline 114 & 0.8 & 0.7 & -0.2 & \\
\hline 115 & 0.3 & 0.3 & 0.0 & \\
\hline 116 & 17.8 & 14.5 & -3.2 & 2.0 \\
\hline 117 & 9.1 & 7.7 & -1.4 & 2.1 \\
\hline 118 & 25.4 & 24.2 & -1.2 & 2.4 \\
\hline 119 & 9.0 & 8.6 & -0.4 & 2.0 \\
\hline 120 & 28.5 & 32.6 & 4.1 & 2.6 \\
\hline 122 & 3.5 & 4.6 & 1.1 & 2.0 \\
\hline 124 & 4.4 & 5.8 & 1.4 & 2.4 \\
\hline Total & 100.0 & 100.0 & $<$ & 2.2 \\
\hline
\end{tabular}

Empty boxes on doubly charged are du to the interference with mail elemental signals. See text for further details

Obviously, to generate quantitative data, one needs matrix-matched reference materials or sensitivity factor models. Limiting on the analytical performance is again the coarse impact of the laser beam with respect to the structure of the thin film. One must, however, note that the large mass of ablated material leads to stoichiometric sampling. Table 2 summarizes sample signal values from the optical laser ablation measurements. Signal values are approx. one order of magnitude higher than those shown below for the XUV laser ablation (Fig. 2), but are (for some isotopes) heavily affected by high blank values. This is a major advantage of XUV laser ablation, since it is not an API technique, but the ionization occurs in vacuum inside the MS.

It was evident that optical laser ablation (laser at $\lambda=532 \mathrm{~nm}$ ) had a coarse spatial resolution for thin-film analysis, and though it provides bulk compositions, these are mixed averages of the various layers. On the other hand, XUV laser ablation mass spectrometry (in the following simply XUV laser ablation) was capable to resolve the spatial structure.

The crater volume, and with that the ablated mass, was determined from the width observed in the electron microscopy (Fig. 1), and the information on the ablated depth obtained by cross-sections shown below. This analysis permitted to estimate five orders of magnitude fewer ablated mass with the XUV laser (label "B" on Fig. 1) as compared to the mass removal done with the $532 \mathrm{~nm}$ system. The resulting mass spectra with XUV laser ablation are shown on Fig. 2, where each panel is a single pulse delivered on top of each other (layer by layer). The $\mathrm{m} / \mathrm{z}$ observed confirms the overall detection capability of the technique as good as 


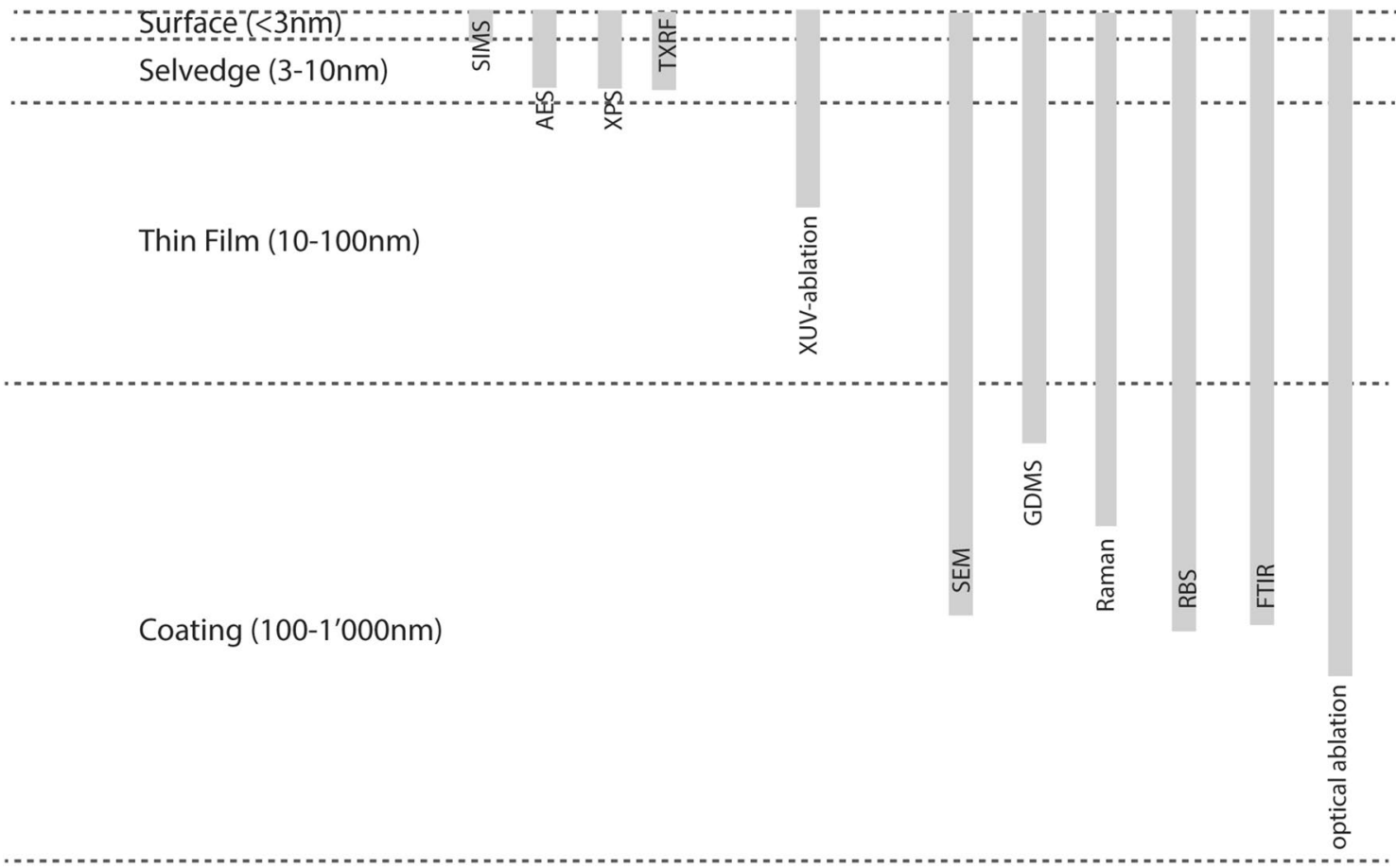

Bulk

Fig. 3 Semi-qualitative depth ranges for a selection of microanalytical techniques along with a nomenclature for structural ranges. Some techniques are static (i.e. the name is insider the bar) and other are dynamic (i.e. the name is below bar), which allows to perform depth profiling by repeated shots. The classification shows that there are techniques for nano-scale analysis which lack the dynamics, or are slow, for direct thin films $(10-100 \mathrm{~nm})$ profiling, while other tech-

it is for optical laser ablation (considering both the signal and the blank).

It is noteworthy to observe that XUV laser ablation was able to spot a $3 \mathrm{~nm}$ top Pt coating (see $\mathrm{m} / \mathrm{z}=194,195,196$ in Fig. 2), intentionally deposited on the surface as marker to check if the depth resolution and sensitivity would allow detecting such fine capping. Such capability is outside the range of state-of-the-art optical laser ablation.

Regarding elemental fractionation, it is not possible to make conclusions from the present data, as this is necessarily assessed using reference materials, which is subject of ongoing research. Nevertheless, the isotopic data obtained, using XUV laser ablation, were well in agreement with theoretical natural abundances (Table 3), within the experimental error of few percent. This permits to exclude any form of isotopic fractionation induced by the short-wavelength laser. It is noteworthy to observe that the deviations, for a given element, cancel each other and sum-up to zero. This indicates that the elemental data are closed, i.e. over-deviations nique are for micro-scale, and are too coarse. AES Auger electron spectroscopy, FTIR Fourier transform infrared, GDMS glow discharge mass spectrometry, RBS Rutherford backscattering spectroscopy, SEM scanning electron microscopy, SIMS secondary ion mass spectrometry, TXRF total (reflection) x-ray fluorescence, XPS X-ray photoemission spectroscopy

counterbalance down-deviations, which may indicate that the observed values are biased by the mass spectrometer.

Whether the use of XUV photons permits to overcome matrix effects, so extensive in other microanalytical techniques, e.g. secondary ion mass spectrometry (SIMS), is still subject of further investigation. The present study also highlighted the occurrence of doubly charged species in XUV laser ablation (Table 3), which was not observed while ablating metallic $\mathrm{Cu}$ samples (not shown in this work). It may be speculated whether the doubly charged are directly sampled from the kesterite as such. In such case, the doubly charged pattern should map the parent element isotopic pattern, i.e. the rate of doubly charged is constant for all isotope of one element. This is the case for Sn (Table 3). If the doubly charged are formed as post-ablation process, the obtained pattern does not have to map the parent element. This is the case for $\mathrm{Cu}$ and $\mathrm{Se}$ (Table 3). The signals of doubly charged $\mathrm{Zn}$ isotopes are largely interfered by main elements in the sample, and were thus not utilized. 


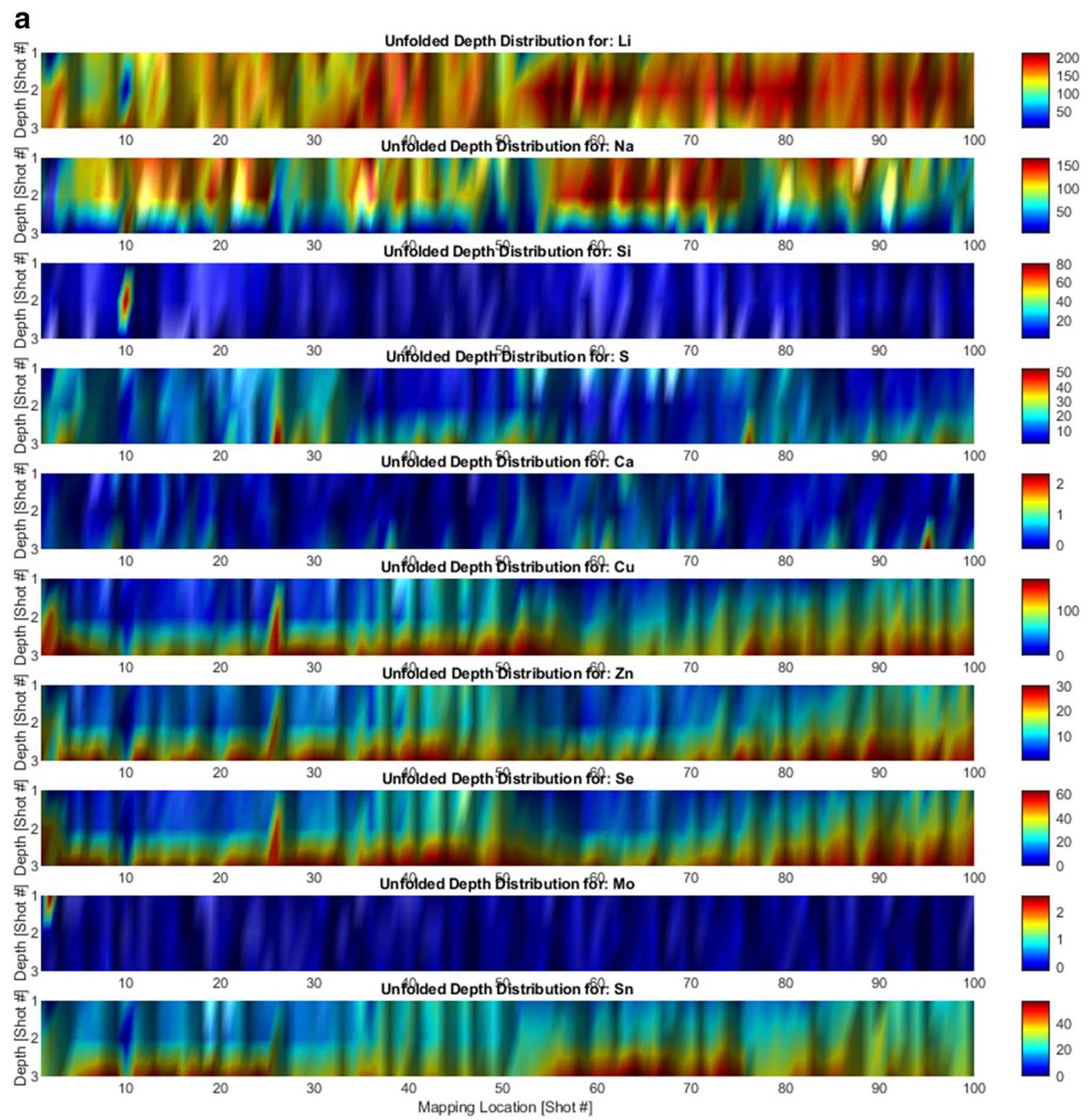

Fig. 4 Depth profiling analysis performed with the XUV-MS, with two different data density resolution. a Chemical inhomogeneity shows superposed to the layered structure; b granular fabric showing the structural heterogeneity. Each stripe provides the 3D distribution of a specific element. The stripe is a map that unfolds the dimension orthogonal to the page along the horizontal axis, with a period of 10

\subsection{Thin-film microanalysis}

With five orders of magnitude fewer sampled mass by the XUV laser, one is surprised to observe a clear signal in the mass spectrum. Since the sensitivity of the two mass spectrometers utilized is not much different, one concludes that the XUV sampling is much more efficient. In fact, both optical laser ablation and XUV laser ablation gave a comparable detection range with much less material ablated in the latter case. Green et al. [11] have estimated the sample utilization units. This explains why the pattern repeats, as the observation is elevated above the page. The vertical scale of each unfolded stripe is the number of repeated shots on-spot. The color scale is the signal intensity in counts, which provides a semi-quantitative information on the abundances

efficiency (SUE) for several microanalytical techniques, such as optical laser ablation, SIMS, and XUV laser ablation. Whatever the efficiency, it can be reasoned that optical laser ablation is a three-step process, i.e. laser vaporization, aerosol transport, plasma vaporization, which makes it prone to losses. On the other hand, XUV laser ablation is a singlestep process, i.e. direct surface ionization, which potentially enhances the SUE. The quantitative assessment of the SUE is beyond the scope of this work, whole focus is to highlight analytical capabilities for the analysis of thin films of 


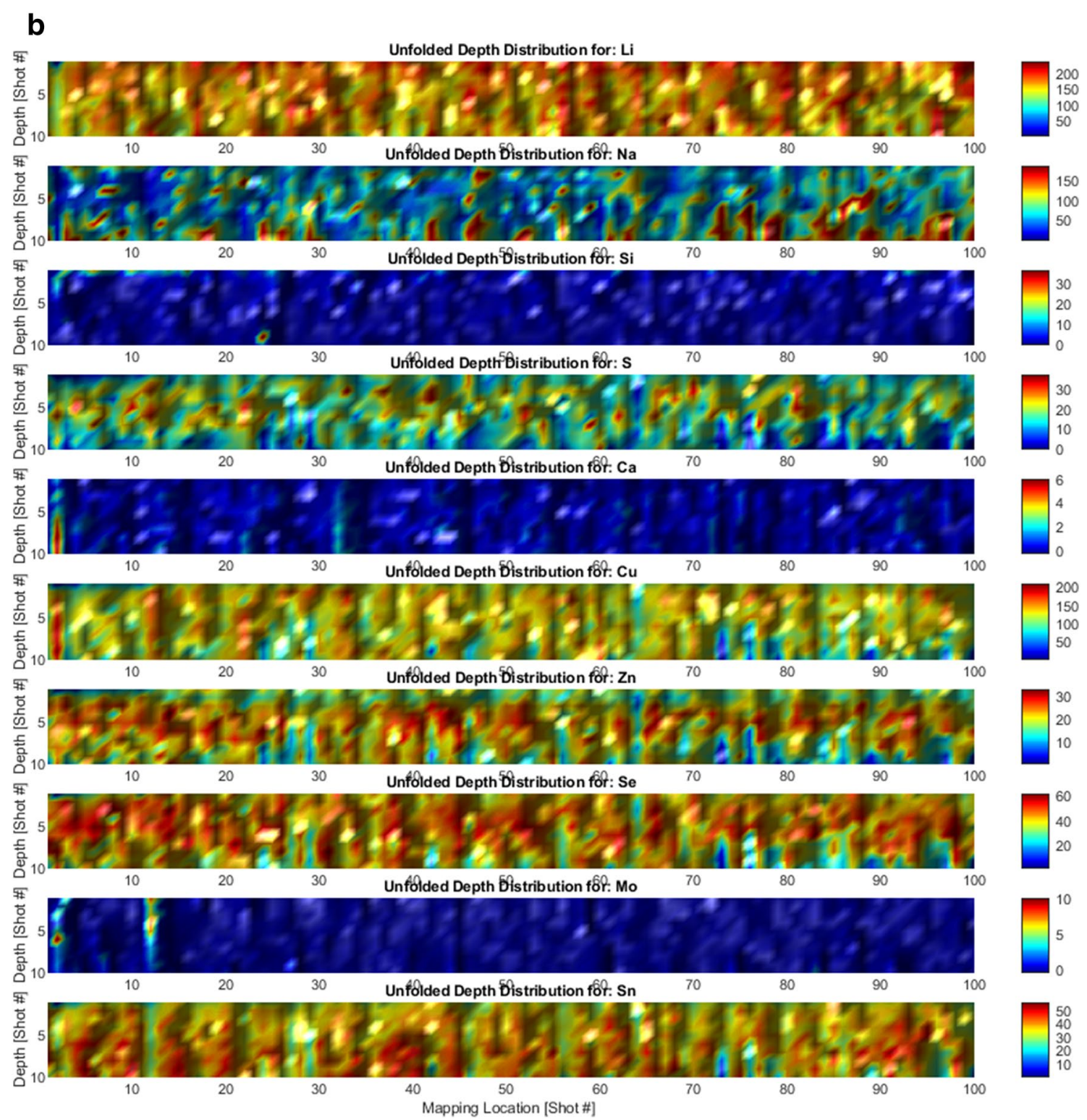

Fig. 4 (continued)

10-100 nm. Indeed, Fig. 3 shows that no state-of-the-art microanalytical technique can directly access such range. Some methods are surface technique, i.e. $<10 \mathrm{~nm}$, whose utilization for thin films is very slow. Other ones are more bulk techniques, e.g. optical laser ablation, and their use for depth resolved analysis of thin films is impractical.

\subsection{Insights into Li-doped photovoltaic thin films}

The data matrix collected with the XUV laser ablation was post-processed to obtain the elemental depth distributions, a selection of which is shown in Fig. 4. The two panel highlight different portion of the sample, analyzed with different data density resolution, one highlighting a well-structured layering (Fig. 4a), the other showing the heterogeneous fabric (Fig. 4b). Data on Fig. 4 permit to observe poor correlation between $\mathrm{Li}$ (alloyed with $\mathrm{Cu}$ ) and $\mathrm{Na}$ in the well-grown portions (Fig. 4a), and the one among $\mathrm{Cu}, \mathrm{Zn}, \mathrm{Se}$, and $\mathrm{Sn}$, as expected. Structural discontinuities are observed. The observed granular fabric of the distributions may be well explained with the porosity of the material. In fact, during the ablation experiments, the signal was unstable, even when normalizing to the laser pulse energy. Figure 5 shows a micrograph of the samples, highlighting the porous fabric, which a previous study put in relation to the content of $\mathrm{Li}$ [4], and the lack of detailed chemical resolution of EDX.

Further observations were made with XUV laser ablation to understand the chemistry of the grown structures. 


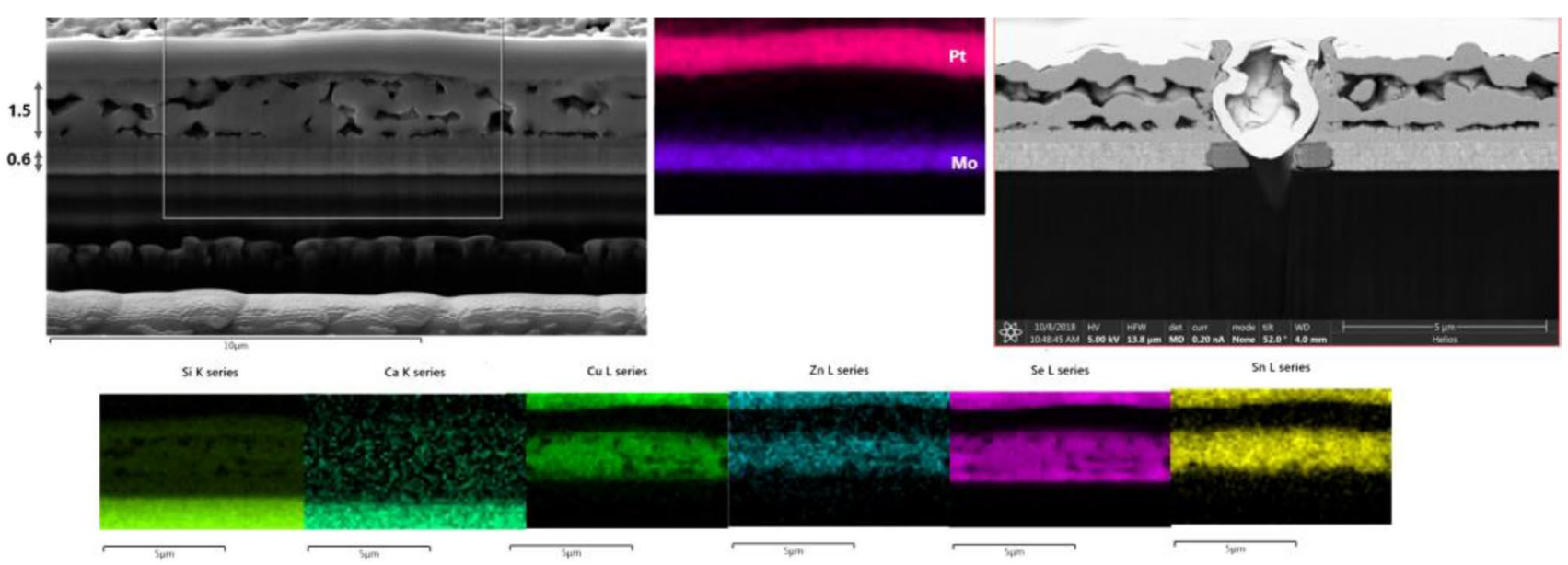

Fig. 5 Scanning electron microscopy (SEM) and energy-dispersive $\mathrm{X}$-rays (EDX), showing the integer stack and one XUV laser ablation crater. The latter was obtained with on-spot repeated irradiation with as many as 100 shots to reach the Mo layer. Pt coating/filling for the micrograph acquisition is shown in white in the laser crater, which was not present during the laser irradiation experiments. In the bottom, the following elemental mappings are shown: $\mathrm{Si}, \mathrm{Ca}, \mathrm{Cu}, \mathrm{Zn}, \mathrm{Se}$, $\mathrm{Sn}$
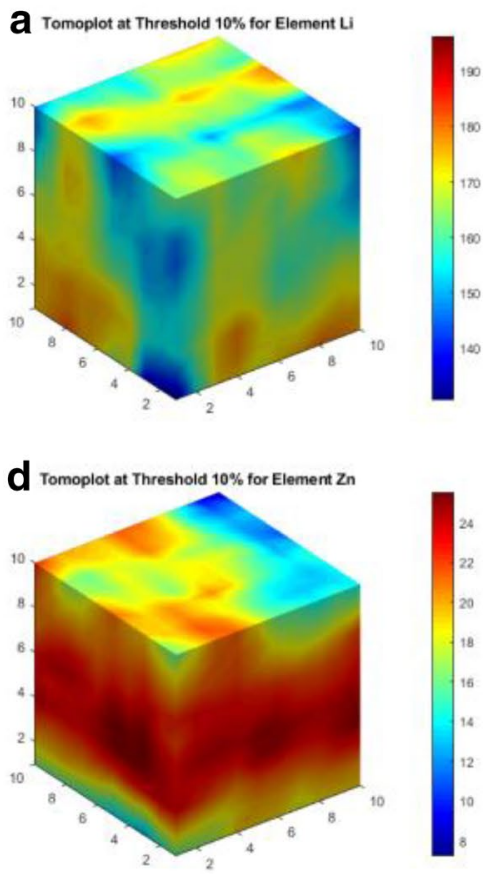

b

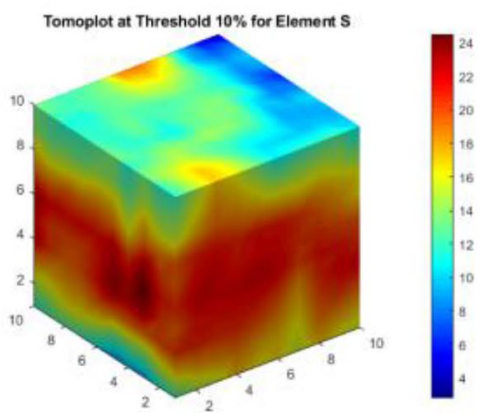

e

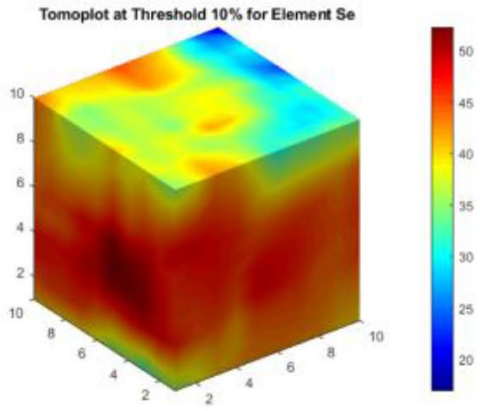

C

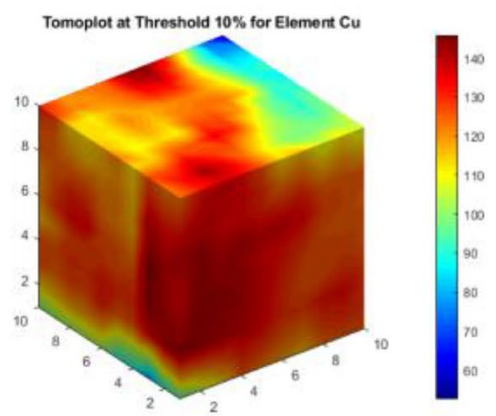

f

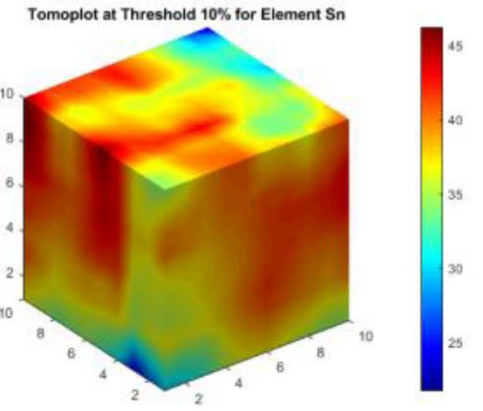

Fig. 6 Block plots of the 3D elemental distribution in regions of $10 \times 10 \times 10$ shots (approx. $10 \times 10 \mu \mathrm{m}$ lateral and $150 \mathrm{~nm}$ in depth as determined from the electron micrographs). One notes the correlation between the chalcophiles and the heterogeneous distribution of the $\mathrm{Li}$ implantation. The color scale is in counts

the crystallographic partitioning of the elements, i.e. elements with similar valence and radius. This can be shown with the aid of block diagrams (Fig. 6). Second, unexpected traces of $\mathrm{Hg}, \mathrm{W}$ and Au were found. These are understood as unwanted contamination in the sputtering chamber where the thin films were prepared. It is important to have such insights to have a better picture of the composition versus 
function relation. In fact, such species may interfere altering the design operation of the PV devices.

\section{Conclusions}

In the present study, the capabilities for depth profiling analysis of thin films by means of laser ablation mass spectrometry have been investigated, based on state-of-the-art optical laser ablation and an advanced XUV laser ablation. The case of Li-alloyed kesterite samples has been studied. The data are semi-quantitative as no external signal calibration was performed to highlight the performance. The plots permit a rapid conversion into quantitative data, once the sensitivity factors are determined with an additional set of calibration run, which was outside the scope of this work.

Considered grain size and film morphology, a $10 \times 10 \mu \mathrm{m}$ map is still small to gather statistically valid patterns. However, the present study has highlighted the limitation of visible lasers to generate a "fine data grid" without sample destruction. The latter was possible, while working with the XUV laser, especially when combined with advanced data processing techniques. Depth distribution maps are, however, robust within the collected data. Here also, the coarse ablation yield of visible lasers did not permit to reconstruct the thin-film structure, which was possible with the XUV laser.

Spatial distribution of Li dopant as well as other elements was reconstructed. As shown, the morphology is inhomogeneous and not compact and same applies to composition. We could not observe segregations or partitioning of the main constituent elements that might suggest the presence of other phases. This aspect is subject of further investigations.

Acknowledgements The authors acknowledge Prof. J. J. Rocca and Prof. C. S. Menoni (Colorado State University) for the original proofof-principle of the Argon capillary discharge laser (now operative in our lab) on our own samples. The research was exclusively funded internally at Empa by the two laboratory involved, as explorative project on instrumentation R\&D.

Open Access This article is licensed under a Creative Commons Attribution 4.0 International License, which permits use, sharing, adaptation, distribution and reproduction in any medium or format, as long as you give appropriate credit to the original author(s) and the source, provide a link to the Creative Commons licence, and indicate if changes were made. The images or other third party material in this article are included in the article's Creative Commons licence, unless indicated otherwise in a credit line to the material. If material is not included in the article's Creative Commons licence and your intended use is not permitted by statutory regulation or exceeds the permitted use, you will need to obtain permission directly from the copyright holder. To view a copy of this licence, visit http://creativecommons.org/licenses/by/4.0/.

\section{References}

1. R. Müller et al., Depth-profiling microanalysis of CoNCN wateroxidation catalyst using a $\lambda=46.9 \mathrm{~nm}$ plasma laser for nanoionization mass spectrometry. Anal. Chem. 90, 9234-9240 (2018)

2. Y. Arbelo, D. Bleiner, Tabletop extreme ultraviolet time-of-flight spectrometry for trace analysis of high ionization energy samples. Rapid Commun. Mass Spectrom. (2019). https://doi.org/10.1002/ $\mathrm{rcm} .8463$

3. J. Koch, M. Wälle, J. Pisonero, D. Günther, Performance characteristics of ultra-violet femtosecond laser ablation inductively coupled plasma mass spectrometry at $\sim 265$ and $\sim 200 \mathrm{~nm}$. J. Anal. At. Spectrom. 21, 932-940 (2006)

4. S.G. Haass et al., Complex interplay between absorber composition and alkali doping in high-efficiency kesterite solar cells. Adv. Energy Mater. 8, 1-9 (2018)

5. A. Cabas-Vidani et al., High-efficiency $\left(\mathrm{LixCu}_{1-\mathrm{x}}\right)_{2} \mathrm{ZnSn}(\mathrm{S}$, $\mathrm{Se})_{4}$ kesterite solar cells with lithium alloying. Adv. Energy Mater. 8, 1801191 (2018)

6. T. Maeda, A. Kawabata, T. Wada, First-principles study on alkali-metal effect of $\mathrm{Li}, \mathrm{Na}$, and $\mathrm{K}$ in $\mathrm{Cu}_{2} \mathrm{ZnSnS}_{4}$ and $\mathrm{Cu}_{2} \mathrm{ZnSnSe}_{4}$. Phys. Status Solidi Curr. Top. Solid State Phys. 12, 631-637 (2015)

7. W. Shockley, H.J. Queisser, Detailed balance limit of efficiency of p-n junction solar cells. J. Appl. Phys. 32, 510-519 (1961)

8. M.A. Green et al., Solar cell efficiency tables (version 53). Prog. Photovoltaics Res. Appl. 27, 3-12 (2019)

9. S. Chen, A. Walsh, X.G. Gong, S.H. Wei, Classification of lattice defects in the kesterite $\mathrm{Cu}_{2} \mathrm{ZnSnS}_{4}$ and $\mathrm{Cu}_{2} \mathrm{ZnSnSe}_{4}$ earthabundant solar cell absorbers. Adv. Mater. 25, 1522-1539 (2013)

10. J. Scragg, J. Wätjen, M. Edoff, A detrimental reaction at the molybdenum back contact in $\mathrm{Cu}_{2} \mathrm{ZnSn}(\mathrm{S}, \mathrm{Se})_{4}$ thin-film solar cells. J Am Chem Soc 134, 19330-19333 (2012)

11. T. Green et al., Characterization of extreme ultraviolet laser ablation mass spectrometry for actinide trace analysis and nanoscale isotopic imaging. J. Anal. At. Spectrom. 32, 10921100 (2017)

Publisher's Note Springer Nature remains neutral with regard to jurisdictional claims in published maps and institutional affiliations. 